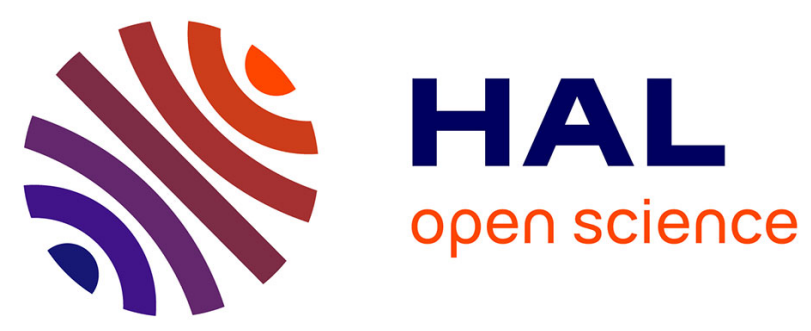

\title{
Former les futurs concepteurs de systèmes homme-machine complexes
}

Hélène Gaspard-Boulinc, Stéphane Conversy, Daniel Prun, Catherine Letondal, Stéphane Chatty

\section{- To cite this version:}

Hélène Gaspard-Boulinc, Stéphane Conversy, Daniel Prun, Catherine Letondal, Stéphane Chatty. Former les futurs concepteurs de systèmes homme-machine complexes. IHM 2013, 25ème conférence francophone sur l'Interaction Homme-Machine, AFIHM, Nov 2013, Bordeaux, France. $10.1145 / 2534903.2534910$. hal-00877248v2

\section{HAL Id: hal-00877248 \\ https://hal.inria.fr/hal-00877248v2}

Submitted on 6 Nov 2013

HAL is a multi-disciplinary open access archive for the deposit and dissemination of scientific research documents, whether they are published or not. The documents may come from teaching and research institutions in France or abroad, or from public or private research centers.
L'archive ouverte pluridisciplinaire $\mathbf{H A L}$, est destinée au dépôt et à la diffusion de documents scientifiques de niveau recherche, publiés ou non, émanant des établissements d'enseignement et de recherche français ou étrangers, des laboratoires publics ou privés. 


\title{
Former les futurs concepteurs de systèmes homme-machine complexes
}

\section{Hélène Gaspard-Boulinc}

Daniel Prun
Catherine Letondal

Stéphane Conversy

Stéphane Chatty

\author{
Université de Toulouse - ENAC \\ 7 avenue Edouard Belin \\ 31055, Toulouse, France \\ prenom.nom@enac.fr
}

\begin{abstract}
RÉSUMÉ
L'ingénierie des systèmes homme-machine implique la définition des besoins des utilisateurs en tant qu'éléments de l'architecture du système. Or l'analyse des pratiques industrielles dans la conception des systèmes complexes met en évidence des problèmes dans la prise en compte de l'utilisateur. Ces problèmes semblent insuffisamment traités par l'apport de cours d'analyse du besoin ou de conception d'IHM. En tant que formateurs de futurs concepteurs de systèmes homme-machine complexes, nous proposons une réponse à ces problèmes par la définition d'un nouvel enseignement en ingénierie des systèmes hommemachine complexes. Cet article présente la conception de cet enseignement, issue d'une réflexion sur les disciplines de l'analyse fonctionnelle, la conception participative et l'approche orientée objet, et menée à la lumière de quatre exigences : définir l'usage, susciter la co-évolution besoin-solution, définir de façon exhaustive les caractéristiques techniques, formaliser les besoins.
\end{abstract}

\section{Mots Clés}

Ingénierie Système; Conception Participative; Conception Orientée Objet; Enseignement.

\section{ACM Classification Keywords}

H.5.m. Information interfaces and presentation (e.g., HCI): Miscellaneous; D.2.1 Requirements/Specifications: Elicitation methods, Methodologies.

\section{INTRODUCTION}

Les systèmes homme-machine posent des défis particuliers aux ingénieurs: prise en compte des « facteurs humains », collaboration avec des métiers utilisant d'autres cadres de référence qu'eux. Pour pallier ces difficultés qui ont mis de nombreux projets en danger, des méthodes de conception adaptées ont été proposées dans les dernières décennies : conception

(C) ACM, 2013. This is the author's version of the work. It is posted here by permission of ACM for your personal use. Not for redistribution. The definitive version was published in Actes de la $25^{\text {ième }}$ conférence francophone sur l'Interaction Homme-Machine, 2013.

http://dx.doi.org/10.1145/2534903.2534910 centrée sur l'utilisateur et conception participative par exemple. Ces méthodes sont enseignées dans nos universités et écoles d'ingénieurs, parfois même font l'objet de formations spécialisées, et elles sont validées par des succès industriels.

Ces méthodes ne répondent pas encore à tous les défis. Ainsi, les diplômés des écoles d'ingénieurs aéronautiques sont destinés à concevoir et réaliser des systèmes complexes : avions, systèmes de contrôle aérien, ou systèmes de gestion d'aéroport. Parmi ces ingénieurs, ceux concernés par l'interaction homme-machine sont une minorité et doivent composer avec des processus d'ingénierie système bien établis, qui constituent le cœur de métier de leur employeur. Par exemple, un avion est constitué de centaines de milliers de composants, conçus et assemblés au cours de milliers de processus gérés de manière systématique. Pour qu'ils puissent exploiter pleinement leurs compétences, il faut espérer à moyen terme l'apparition de processus d'ingénierie de systèmes homme-machine compatibles d'une part avec les méthodes d'ingénierie système établies, et de l'autre avec les méthodes de conception de systèmes interactifs.

Des travaux tels que [2] [33] [11] proposent des méthodes intégrant l'IHM dans les processus de développement logiciel. Cependant, il est difficile pour les concepteurs d'IHM d'exiger que la mécanique bien huilée des industriels s'adapte à de nouvelles méthodes, si cela doit mettre en danger les autres processus de conception et de production.

Néanmoins il est possible d'agir à court terme sans attendre que les méthodes d'ingénierie de systèmes homme-machine soient assez mûres pour être systématisées par les grands industriels. En effet, si l'on parvient à former des ingénieurs qui maîtrisent les différentes méthodes, comprennent leurs relations, voire disposent de techniques pour les combiner, alors ces ingénieurs devraient trouver plus facilement leur place dans les processus industriels et être capables de proposer de meilleurs compromis. L'intégration des différentes approches relève alors de la responsabilité de nos jeunes ingénieurs lorsqu'ils arrivent en entreprise, en l'absence de méthode "intégratrice » commune à tous les employeurs. L'enjeu associé est double: aider les 
grands systèmes aéronautiques à absorber dans les prochaines années les nécessaires innovations en matière d'interaction homme-machine, et progresser vers la prise en compte des humains comme des composants à part entière de ces systèmes complexes.

Cet article décrit une expérience pédagogique dans ce sens, évaluée informellement à travers les résultats des élèves ingénieurs de deux promotions successives et la perception de leurs prestations par les "clients". Nous commençons par analyser les problèmes de l'ingénierie des systèmes homme-machine, en termes de pratiques industrielles et d'enseignement. Nous en déduisons des objectifs pédagogiques à atteindre pour la formation des futurs ingénieurs. Nous identifions ensuite trois méthodes actuellement proposées en ingénierie système ou en ingénierie des interfaces homme-machine : analyse fonctionnelle, analyse " orientée-objet» et conception participative. Nous décrivons alors en détail la manière dont la pédagogie proposée combine ces trois méthodes. Nous proposons enfin un retour d'expérience sur l'application de l'enseignement par les étudiants. Nous concluons en discutant la validité des résultats observés.

\section{L'INGENIERIE DES SYSTEMES HOMME-MACHINE}

Les systèmes homme-machine se caractérisent par la présence d'utilisateurs en interaction avec le système technique, soit comme opérateurs, soit comme superviseurs de décisions prises par la machine. L'ingénierie des systèmes homme-machine implique donc la définition des besoins des utilisateurs en tant qu'éléments de l'architecture du système.

\section{Analyse des pratiques industrielles}

Les processus dits d' " ingénierie système » sont adoptés comme cadre méthodologique par les grandes entreprises aéronautiques, employeurs des étudiants que nous formons. L'ingénierie système est une approche collaborative et interdisciplinaire dont l'objectif est de définir, concevoir, réaliser et maintenir des systèmes complexes, en cherchant à élaborer une solution optimisée en performance, coût et délai. Au sein de cette démarche normalisée, le processus «stakeholder requirement definition » a comme objectif principal la définition des besoins qu'un système doit satisfaire afin de fournir un ou plusieurs services à une ou plusieurs parties prenantes, dans un environnement spécifique [18].

Les analyses des projets de conception de systèmes dans le monde comme celle du Standish Group [34], font état d'un taux de réussite (projet livré à temps, dans le budget et réalisant toutes les caractéristique demandées) de l'ordre de $30 \%$, d'un taux d'échec (arrêt du projet) de l'ordre de $20 \%$ et d'un taux de projet partiellement réussi (mené à terme et opérationnel mais hors budget, et/ou hors délai et/ou ne répondant pas à toutes les caractéristiques demandées) de l'ordre de 50\%. Les causes identifiées de ces échecs sont majoritairement liées à l'identification des besoins des parties prenantes : manque d'entrées de l'utilisateur, incomplétude de la spécification, évolution tardive des spécifications. Face à ce constat, les analyses des processus actuels d'ingénierie des systèmes complexes dans [5] et [21] préconisent notamment une meilleure intégration de l'utilisateur dans ces processus et une plus grande agilité dans la conduite des processus. Cependant, cela reste à l'état de préconisation et d'axes de recherche, sans proposer pour l'instant de solutions opérationnelles pour les industriels. Une des difficultés est que chaque entreprise a développé ses propres pratiques d'ingénierie, et qu'il est donc difficile de définir des solutions applicables par tous. D'autre part, une résistance au changement au sein des entreprises est constatée par [21], notamment au niveau des personnes responsables de la gestion des marchés, avec le syndrome du THWADI («That's how we've always done it»). La philosophie prédominante du cycle en $\mathrm{V}$ implique une linéarité séquentielle spécification-réalisation-tests qui est favorable (en apparence) aux relations contractuelles.

\section{Analyse de nos enseignements}

Dans les curriculums actuels d'ingénierie $[15,16]$, trois disciplines différentes proposent des processus et des techniques pour la définition des besoins des utilisateurs : l'interaction homme-machine, l'ingénierie système et le génie logiciel. Nous dispensions jusqu'en 2010 des cours différenciés d'Ingénierie Système, de Conception Participative de système interactifs, et de Conception Orientée-Objet. Ces enseignements abordaient tous la problématique de collecte des besoins et de conception, avec des points de vue différents. Nous avons constaté que, bien qu'ayant compris et appris à maîtriser les démarches proposées par chacune de ces disciplines, les étudiants avaient des difficultés à comprendre qu'elles participaient toutes au même processus de définition des besoins. Ces difficultés se révélaient lors des projets d'étudiants et des stages de fin d'études: prise en compte souvent symbolique des utilisateurs («j'ai recueilli les besoins auprès de mon maitre de stage »), méthodes de collecte inadaptées ( «'ai fait une analyse en mettant un questionnaire en ligne $»)$, exigences et critères d'évaluation flous ( «j'ai fait un logiciel intuitif »). Les étudiants faisaient également part de leur difficulté à appliquer les méthodes enseignées lorsqu'ils étaient en contexte industriel, et préféraient alors oublier leurs cours en faveur d'une meilleure collaboration avec leur encadrement industriel.

\section{Exigences d'un nouvel enseignement}

En tant que formateurs des futurs concepteurs des systèmes homme-machine complexes, nous proposons de reprendre ces analyses des pratiques industrielles et des enseignements, afin de répondre aux problèmes par la définition d'un nouvel enseignement. En effet, l'analyse de l'existant montre que l'ingénierie système pratiquée par les industriels pour la conception de systèmes complexes a des bonnes propriétés qu'il faut conserver, relatives à la gestion de la complexité : la recherche d'une exhaustivité sur les caractéristiques techniques du système, et la 
formalisation du besoin. Ce sont les deux premières exigences auxquelles doit répondre un enseignement en ingénierie des systèmes homme-machine complexes. Cette analyse met également en évidence des problèmes liés à une mauvaise prise en compte des utilisateurs dans la conception du système, et une évolution des besoins des utilisateurs dans le temps. Les enseignements actuels en conception participative et en conception orientée objet proposent des solutions pour traiter ces problèmes, en proposant de définir l'usage du système à concevoir et de susciter la co-évolution besoin-solution avec un processus itératif. Ce sont deux autres exigences auxquelles doit répondre le nouvel enseignement. La figure 1 présente les objectifs pédagogiques de l'enseignement d'ingénierie des systèmes hommemachine complexes, qui reprend ces exigences.

Dans le cadre d'un projet de conception ou d'évolution d'un système homme-machine complexe, à partir d'une demande ou d'un cahier des charges préliminaire, l'étudiant saura collecter et formaliser les besoins. On peut décliner ces objectifs en sous-objectifs suivants.

- Susciter la co-évolution besoin-solution :

- savoir justifier les ressources nécessaires à la collecte des besoins auprès des utilisateurs ;

- savoir citer des techniques et notations pour la collecte des besoins des utilisateurs;

- savoir mettre en auvre certaines de ces techniques et notations ;

- Définir l'usage :

- savoir justifier l'importance de définir l'usage du système;

- savoir citer des techniques et notations pour définir l'usage du système;

- savoir mettre en cuvre certaines de ces techniques et notations,

- Décrire de façon exhaustive les caractéristiques techniques:

- savoir citer des techniques et notations pour la recherche exhaustive des caractéristiques techniques du système;

- savoir mettre en cuvre certaines de ces techniques et notations système;

- Formaliser :

- savoir citer des techniques et notations pour l'expression des besoins ;

- savoir mettre en cuvre certaines de ces techniques et notations ;

- savoir rédiger un document d'expression des besoins ,

- savoir utiliser ce document dans la relation client-fournisseur.

Figure 1 : les objectifs pédagogiques de l'enseignement

A travers la distinction entre cahier des charges préliminaire en entrée, et document d'expression des besoins en sortie, nous mettons en avant le fait que le cahier des charges rédigé par un client sans étude préalable peut être incomplet et insuffisant pour établir un contrat avec un fournisseur. La collecte et l'expression des besoins nécessitent des compétences spécifiques, qui sont rarement maitrisées en interne par le client. Et si elles le sont, il reste difficile de mener plusieurs rôles qui souvent se cumulent : utilisateur, acquéreur, chef de projet. Nous souhaitons insister auprès des étudiants sur le fait qu'un des rôles de l'ingénieur est d'accompagner la découverte et la formalisation des besoins.

A ce stade, nous ne précisons volontairement pas les techniques et notations enseignées pour atteindre ces objectifs : celles-ci sont en constante évolution, et nous ne souhaitons pas asservir les objectifs à telle ou telle approche, mais au contraire rester le plus ouvert possible à toute nouvelle technique qui prouverait son efficacité dans le futur. C'est cette démarche qui a guidé Coad et Yourdon [10] dans la définition de l'analyse orientée objet, et que nous nous proposons de suivre, en analysant les forces et les limites de l'analyse fonctionnelle, la conception participative et l'analyse orientée objet.

\section{TROIS DISCIPLINES, TROIS METHODOLOGIES}

\section{L'analyse fonctionnelle}

\section{Présentation}

En ingénierie des systèmes, l'analyse fonctionnelle désigne un ensemble de méthodes permettant de mener à bien le processus de «stakeholder requirement definition $»$. On peut citer par exemple les plus connues : SAFE (Sequential Analysis of Functional Elements), FAST (Functional Analysis System Technique) [7], SADT (Structured Analysis Design Technique) [32], APTE [13], KAOS [36], GRL [20]. Ces méthodes proposent une approche basée sur l'étude des fonctions ou des buts [28]. Une fonction décrit une transformation d'entrée en sortie qui peut correspondre à la création, surveillance, modification ou destruction d'éléments, voire une transformation nulle. Un but décrit un effet recherché, un objectif devant être atteint. L'idée directrice sousjacente de cette approche est que la connaissance des fonctions ou des buts découlant d'un besoin aide à mieux définir le produit qui satisfera ce besoin.

Largement utilisée, l'analyse fonctionnelle permet de contribuer à une meilleure caractérisation du besoin, en proposant une démarche rationnelle et systématique de recherche, de caractérisation et de classification des fonctions. Par exemple la méthode APTE propose une démarche structurée en quatre étapes conduisant à l'identification des fonctions à réaliser, des contraintes à prendre en compte pour réaliser ces fonctions ainsi que des diverses caractéristiques non fonctionnelles associées telles que la performance ou encore la capacité des fonctions (les critères de valeurs).

\section{Limites}

Dans le contexte des systèmes homme-machine complexes, les méthodes d'analyse fonctionnelle présentent cependant certaines limites. D'une part, il y a 
l'hypothèse tacite qu'elles sont menées par un « ingénieur système » responsable du processus d'élaboration des besoins, mais sans préciser l'implication des diverses autres parties prenantes, en particulier celle de l'utilisateur final. Il en est de même pour les différentes normes associées: l'ISO 15288 fait référence à la norme ISO 18152, mais cette dernière ne donne pas de méthode effective. Le SE Handbook [17] se contente de citer les méthodes suivantes « interviews, focus groups, the Delphi technique and soft system methodology » sans fournir davantage d'information sur leur mise en œuvre.

D'autre part, les parties prenantes ont des besoins qui concernent moins les aspects fonctionnels que les caractéristiques non fonctionnelles du système. L'utilisabilité est devenue centrale pour beaucoup de systèmes au delà de la seule utilité (caractérisée par les fonctions offertes). Les approches d'ingénierie système «orientées fonctions» ne sont par conséquent pas suffisantes pour capturer et exprimer l'ensemble du besoin, même si certaines offrent des possibilités d'aborder les aspects non fonctionnels du besoin mais sans préciser comment traiter l'utilisabilité (cf. les critères de valeurs de la méthode APTE).

Enfin, les méthodes d'analyse fonctionnelle font l'hypothèse que le besoin peut être identifié avant toute réalisation du système final. Elles se placent dans un cycle de vie classique identifiant d'abord une étape de spécification du besoin suivie d'une étape de recherche d'une solution à ce besoin. Or Boehm [5] note que « les exigences pour les systèmes interactifs ne sont généralement pas pré-spécifiables à l'avance, mais émergent avec l'usage ». La logique de l'approche fonctionnelle séparant le problème (le quoi ?) de la solution (le comment? l'usage ?) doit donc être revue au profit d'une approche basée sur la co-évolution entre le besoin et sa réalisation [6].

\section{La démarche de Conception Participative}

\section{Présentation}

Les démarches de conception de systèmes interactifs ont pour but de produire des systèmes utilisables, c'est-à-dire qui permettent aux utilisateurs de mener à bien leurs activités de façon efficace et stimulante. Les systèmes interactifs sont difficiles à définir en terme de solutions à des problèmes bien définis, et cela constitue une des difficultés des projets de développement de ces systèmes. Leur caractère fondamentalement évolutif [8], voire coévolutif [23] vient à la fois de l'imprévisibilité des usages de ces systèmes [11] et d'une inter-dépendance intrinsèque entre les « solutions » qu'ils apportent et les «problèmes » qu'ils résolvent. Afin de pallier cette difficulté, la dernière norme internationale sur la conception de systèmes interactifs recommande la participation active et fréquente des utilisateurs durant toutes les phases de conception [19]. La conception participative (CP) est une méthode qui s'appuie sur la participation des utilisateurs, non seulement durant les phases d'analyse et d'évaluation, mais aussi durant celles d'idéation et de conception [29].

La CP considère que les interfaces (frontière entre homme et machine) ne sont qu'une partie du résultat de la conception. L'objectif est moins d'instrumenter une activité existante avec une nouvelle interface, que de réinventer une activité, ses finalités, et une instrumentation adaptée. Ainsi la conception est centrée activité, et plus seulement centrée utilisateur [30]. Cet objectif implique que la conception s'inscrit intrinsèquement dans la durée : les concepteurs doivent s'approprier le problème, et travailler de façon itérative en alternant les phases d'exploration de solutions, et les phases d'intégration dans un système cohérent, détaillé et concret. Cet objectif implique aussi que les méthodes de conception mises en œuvre ne se contentent pas d'intégrer les concepteurs dans le monde des utilisateurs, mais qu'elles intègrent les utilisateurs dans le monde des concepteurs afin que ces derniers soient parties prenantes de la définition des solutions. Ainsi, au lieu de regretter " que les besoins changent tout le temps», les acteurs d'une démarche de CP admettent et suscitent leur évolution, et organisent leur façon de travailler autour du principe de la coévolution de la compréhension du problème et de la conception de la solution.

\section{Limites}

Cependant, bien que cela soit proposé explicitement par certaines approches comme celle du Contextual Design [3], la littérature classique de l'enseignement de la CP [25] aborde peu la formalisation des besoins, qui est pourtant nécessaire à une bonne communication entre les différentes équipes de développement. Les retours des industriels lors des conseils de perfectionnement de nos formations confirment que les diplômés ne sont pas suffisamment aguerris à la rédaction de synthèse, ce qui rend difficile l'exploitation des résultats par les phases « aval» du processus de conception. Par ailleurs, les enseignements en CP insistent sur la maîtrise de techniques (interview, scénario de travail, brainstorming [24]), mais abordent peu la conduite globale du processus: par exemple, peu de cours sont consacrés à la façon d'organiser de façon systématique le processus afin d'aborder l'ensemble des problèmes à résoudre, d'accorder des priorités à des fonctions à réaliser, ou de gérer les différentes spécialités des acteurs de la conception (ergonomes, designers graphiques, etc.).

\section{L'approche orientée objet}

\section{Présentation}

L'approche orientée objet répond d'abord à un besoin de rééquilibrer l'aspect fonctionnel par un aspect « données », complémentaire. Comme le montraient déjà Coad et Yourdon [10] la modélisation du domaine par les « objets», c'est-à-dire par l'identification des concepts pertinents du domaine et de leurs relations, apporte une dimension stabilisante à l'analyse du problème et des besoins. Dans le contexte d'une activité, 
les concepts désignant les objets du domaine sont en effet moins amenés à changer que les fonctions du système, on peut même dire que les objets sont les composants les plus réutilisables d'une application [27]. Les objets s'avèrent aussi un bon support de communication avec les experts du domaine, car ils représentent le contexte de l'activité à un niveau d'abstraction familier et pertinent pour eux. Les concepts analysés peuvent ensuite être réutilisés lors des phases de conception en conjonction avec les cas d'utilisation du système, pour allouer des responsabilités aux objets correspondant à ces concepts.

\section{Limites}

Si l'approche orientée objet apporte une formalisation des intentions métiers à travers les cas d'utilisation, elle manque en revanche de moyens concrets et efficaces pour détecter et déterminer ces intentions métiers [31]. L'identification des cas d'utilisation ne repose pas suffisamment sur une réelle compréhension de l'activité et de son évolution. D'autre part, la notation UML, associée généralement à l'approche orientée objet, peut représenter un problème dans la communication avec les utilisateurs. Des projets tels que [9] ayant combiné l'approche objet et la conception participative suggèrent que la modélisation du domaine peut être efficacement approchée par un travail coopératif avec les utilisateurs concernés, en utilisant non pas la notation UML mais une représentation préliminaire informelle et non exhaustive.

Nous rencontrons également une limite dans l'utilisation de l'analyse orientée objet pour la conception de systèmes très interactifs, du fait de l'abstraction exigée par l'analyse conceptuelle, qui conseille d'écarter toute interface concrète et tout interacteur des concepts du domaine. Lorsqu'il s'agit d'un système fortement interactif, ce précepte résulte en un fossé important entre le modèle du domaine, par définition éloigné du fonctionnement du système à concevoir, et les interactions conçues notamment en s'appuyant sur le prototypage produit lors des phases de conception participative. Une possibilité serait de considérer les interacteurs comme des "agents » interagissant avec le système, mais cette solution n'est pas satisfaisante car elle écarte les interacteurs des concepts centraux du système. Ainsi, les architectures en couche ne laissent qu'un rôle très restreint aux éléments de l'interface dans la sémantique du système. Il en résulte souvent un décalage entre le modèle du domaine et le modèle de conception qui comporte essentiellement des classes IHM, s'appuyant en général sur l'une ou l'autre des variantes du modèle MVC [35], et qui réutilise très peu les concepts du domaine déterminés lors de l'analyse.

\section{UN ENSEIGNEMENT D'INGENIERIE DES SYSTEMES HOMME-MACHINE COMPLEXES}

A partir de l'analyse réalisée des différentes disciplines existantes, notre démarche vise à concevoir un enseignement en ingénierie des systèmes homme- machine complexes, par une combinaison qui permet de compenser les limites d'une discipline par les points forts d'une autre.

La figure 2 synthétise les points forts et les limites des disciplines présentées précédemment, selon les quatre principales exigences identifiées en termes de compétences pour les futurs concepteurs. On peut voir sur cette figure qu'une combinaison adéquate des différentes disciplines pourrait nous permettre de remplir nos exigences. La combinaison conçue se fait selon des principes en termes de processus, techniques et productions.

\section{Processus}

Les processus d'ingénierie système sont pris comme cadre de référence. En effet, les processus IS sont les plus utilisés par les futurs employeurs, et les plus larges : nous formons des concepteurs de systèmes complexes, et non des concepteurs de logiciels interactifs. Cependant, nous insistons auprès des étudiants pour une application non linéaire de ces processus, en préconisant des itérations (exigence « susciter la co-évolution besoin-solution »).

\section{Techniques}

Nous retenons les techniques issues des différentes disciplines, qui répondent à nos exigences, en montrant leur intégration dans les processus d'ingénierie système, et leur complémentarité.

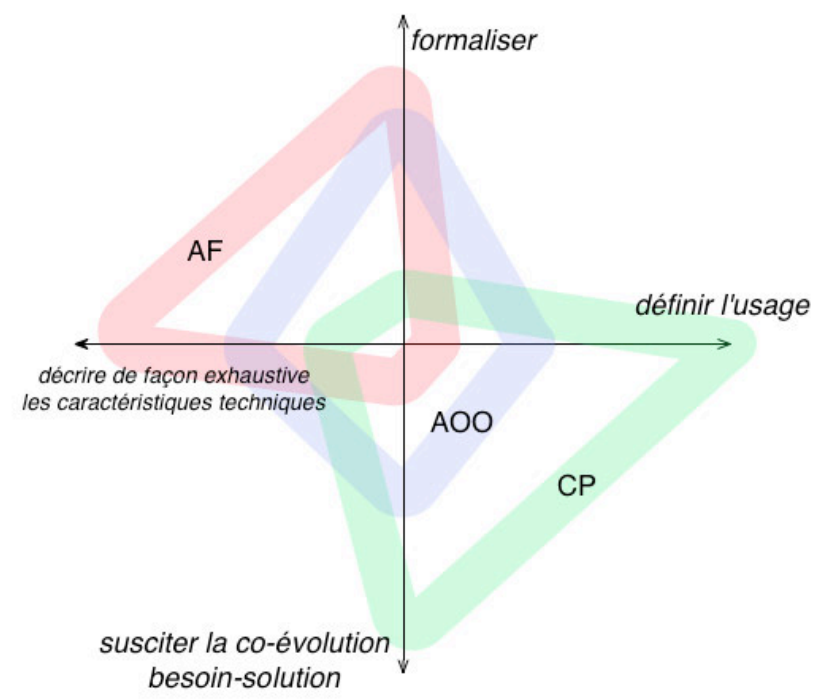

Figure 2 : synthèse des points forts et limites des disciplines AF, CP et AOO selon les exigences identifiées

Les étudiants sont formés à la technique de l'enquête contextuelle $(\mathrm{CP})$ pour la collecte des besoins (exigence « définir l'usage »), et doivent être capables de justifier l'utilisation de cette technique par rapport à des réunions classiques avec le client ou des représentants d'utilisateurs.

Cette technique est complémentaire de la méthode APTE (AF), enseignée pour une recherche systématique 
des fonctions et de leurs critères de valeur (exigences "définir de façon exhaustive les caractéristiques techniques du système» et « formaliser »), en permettant d'identifier et affiner les parties prenantes en interaction avec le système technique (ou éléments du milieu extérieur dans la terminologie APTE), et d'élargir l'enquête contextuelle à des questions sur les performances du système.

La rédaction de scénario est enseignée, d'une part comme technique de restitution des enquêtes contextuelles avec les scénarios de travail (CP), et d'autre part comme technique d'expression des besoins avec les cas d'utilisation (AOO). La déduction des cas d'utilisation du système à concevoir à partir des scénarios de travail permet de s'assurer du respect des intentions métier des utilisateurs et de mettre l'accent sur l'usage du système (exigence « définir l'usage »).

La technique de brainstorming avec les parties prenantes est préconisée en conception participative, mais également en analyse fonctionnelle pour l'identification des fonctions et des événements redoutés, utiles pour les études de sûreté de fonctionnement. Nous enseignons cette technique, en insistant sur l'intérêt d'y inclure les utilisateurs pour compléter la collecte des besoins (exigence «susciter la co-évolution besoinsolution»), et travailler sur l'usage et la dynamique du système futur (exigence «définir l'usage »), avec la technique du brainstorming vidéo (CP).

$\mathrm{La}$ technique de rédaction des besoins unitaires $(\mathrm{AF} / \mathrm{IS})$ est enseignée, avec des règles syntaxiques et grammaticales à respecter. Cette technique permet d'exprimer de façon la moins ambiguë possible les besoins de façon unitaire (exigence « formaliser »), et de mettre au même niveau les besoins fonctionnels et les besoins non fonctionnels, notamment ceux liés à l'utilisabilité et à l'usage (exigence « définir l'usage»). Ainsi chaque cas d'utilisation fait l'objet d'un besoin unitaire de type « le système doit permettre de réaliser le cas d'utilisation $\mathrm{XX} »$

Nous enseignons les patterns de conception GRASP [22] (AOO) qui consiste à concevoir les classes par la réalisation des cas d'utilisation dans des diagrammes de séquence de conception, par lequel le concepteur peut visualiser précisément les interactions entre objets et en déduire des propriétés générales de qualité logicielle, telles que la cohésion, ou le faible couplage. Cette méthode permet, par la réutilisation des concepts du domaine et des cas d'utilisation, de faire un lien explicite entre les analyses objet et fonctionnelle, la conception participative et la conception concrète des composants logiciels dans leur dimension dynamique.

\section{Productions}

Le résultat de la conduite des processus et de l'application des techniques décrites précédemment est un document d'expression des besoins (exigence « formaliser »), dont nous avons établi la trame à partir du modèle de cahier des charges fonctionnel de la norme [1], et que nous élargissons à des aspects non fonctionnels (exigence « définir l'usage »).

La notion de scénario est centrale dans ce document. Nous proposons un modèle de scénario de travail (CP), guidant les étudiants dans une analyse systématique du scénario, avec une explicitation des points forts et des points faibles mis en évidence dans la description textuelle et les photos/schémas (cf. figure 3).

Nous proposons également un tableau (cf. figure 3), reprenant les points forts et faibles identifiés, avec une transformation en besoins unitaires. Cela permet d'assurer la traçabilité entre besoins exprimés et enquêtes contextuelles, via les scénarios, afin de justifier l'existence des besoins issus de l'usage (exigence « définir l'usage »).

\begin{tabular}{|l|l|}
\hline Titre & Consultation de l'emploi du temps depuis les bornes \\
Intérêt & Montrer les habitudes d'utilisation des utilisateurs \\
Qui & 2 élèves d'IENAC \\
Où & ENAC, couloir \\
Contexte activité & 1 minute avant le début des cours \\
Contexte système & Borne \\
\hline
\end{tabular}

\begin{tabular}{|l|l|}
\hline Description & + Points forts/ -Points faibles \\
\hline $\begin{array}{l}\text { L'utilisateur sélectionne le nom de sa } \\
\text { promotion avec la trackball ou la } \\
\text { souris dans le menu déroulant : } \\
\text {-II clique sur IENAC, puis IENAC11, } \\
\text { puis sa filière. }\end{array}$ & $\begin{array}{l}\text { (-) Se réinitialise à la promo par défaut } \\
\text { au bout d'un certain temps (perte de } \\
\text { temps puisqu'il faut refaire les même } \\
\text { manipulations). } \\
\text { (-) Menus déroulant désagréable et } \\
\text { peu performant. } \\
\text { (+) Possibilité d'entrer le nom de sa } \\
\text { promotion dans la barre de recherche. }\end{array}$ \\
$\begin{array}{ll}\text { L'utilisateur sélectionne la semaine } \\
\text { courante en faisant défiler un menu } \\
\text { horizontal. }\end{array}$ & $\begin{array}{l}\text { (-) Affichage d'une semaine par défaut } \\
\text { (perte de temps). } \\
\text { (-) Si l'utilisateur se trompe et clique } \\
\text { sur l'icône en forme de croix le } \\
\text { système affiche la semaine par défaut } \\
\text { et la semaine sélectionnée : } \\
\text { I'affichage devient illisible. }\end{array}$ \\
\\
\end{tabular}

\begin{tabular}{|l|c|l|}
\hline \multicolumn{1}{|c|}{ Points faibles } & Issu du scénario & \multicolumn{1}{c|}{ Besoin } \\
\hline $\begin{array}{l}\text { Le système est initialisé à la } \\
\text { promotion EPL par défaut. }\end{array}$ & 1 & $\begin{array}{l}\text { Le système initialise l'emploi du } \\
\text { temps à la promotion de } \\
\text { I'utilisateur qui se logue. }\end{array}$ \\
\hline $\begin{array}{l}\text { Le système se réinitialise à la } \\
\text { promotion par défaut au bout } \\
\text { d'un certain temps. }\end{array}$ & 1 & $\begin{array}{l}\text { Le système ne se réinitialise pas en } \\
\text { cours d'utilisation. }\end{array}$ \\
\hline $\begin{array}{l}\text { Le système affiche la même } \\
\text { semaine par défaut quelque soit la } \\
\text { semaine courante et parfois } \\
\text { n'affiche qu'un seul jour de cette } \\
\text { semaine. }\end{array}$ & 1 & $\begin{array}{l}\text { Le système s'initialise à la semaine } \\
\text { en cours par défaut et sélectionne } \\
\text { tous les jours de cette semaine. }\end{array}$ \\
\hline
\end{tabular}

Figure 3 : modèle de scénario de travail et transformation en besoins 
Par rapport à des formalismes nécessitant un apprentissage, tels que les réseaux de Petri, les maquettes papier produites lors des brainstormings ont l'avantage d'être compréhensibles et manipulables directement par les utilisateurs (exigence « susciter la co-évolution besoinsolution»). Leur inconvénient est leur manque de précision, car bien qu'elles soient ensuite utilisées comme entrée à la rédaction des cas d'utilisation, elles restent des supports difficilement exploitables par des concepteurs n'ayant pas participé aux séances. Aussi les diagrammes de séquence système, utilisés en analyse orientée objet, permettent de formaliser la dynamique du système à partir des scénarios de conception, et d'assurer l'utilisation de ces résultats dans la suite du processus de conception du système (exigences « formaliser » et « définir l'usage »).

Enfin, un tableau de synthèse des besoins est proposé (cf. figure 4). L'intérêt de cette synthèse est multiple. Pour la spécification et l'établissement d'un contrat entre client et fournisseur, elle propose une vision globale et formalisée des besoins. Elle constitue une base intéressante pour l'établissement du référentiel des exigences. En effet, mieux les besoins sont exprimés, plus le passage du besoin (langage utilisateur) aux exigences (langage du concepteur) est aisé (exigence « formaliser »). Pour le management de projet, les priorités validées par les parties prenantes permettront de définir le contenu des itérations. Pour la conception, l'utilisation de la méthode de conception pilotée par les responsabilités [22], permet de poursuivre l'intégration des différentes disciplines. En effet, dans cette méthode, la conception détaillée des diagrammes de classes s'effectue par une synthèse des scénarios, des fonctions et des données. La réalisation des cas d'utilisation, qui sont issus à la fois des scénarios de conception de la CP et de l'analyse fonctionnelle, repose sur l'utilisation du modèle du domaine, qui sert donc de vecteur pour guider l'allocation rationnelle de « fonctions » aux composants logiciels.

\begin{tabular}{|c|c|c|c|}
\hline Identifiant & Besoin & Justification & $\begin{array}{l}\text { Niveau de priorité } \\
\text { (obligatoire, } \\
\text { secondaire, } \\
\text { optionnel) } \\
\end{array}$ \\
\hline B1 & \multicolumn{3}{|l|}{ Services } \\
\hline B1.1 & $\begin{array}{l}\text { Le système doit permettre } \\
\text { aux élèves de prendre } \\
\text { connaissance de l'edt. }\end{array}$ & AFE & obligatoire \\
\hline B1.2 & $\begin{array}{l}\text { Le système doit permettre } \\
\text { la mise à jour de l'edt par } \\
\text { le bdp. }\end{array}$ & AFE & obligatoire \\
\hline B1.2 & $\begin{array}{l}\text { Le système doit permettre } \\
\text { de sélectionner la } \\
\text { semaine d'edt. }\end{array}$ & AFE/Storyboard & obligatoire \\
\hline B1.4 & $\begin{array}{l}\text { Le système doit permettre } \\
\text { d'envoyer par mail un edt. }\end{array}$ & AFE/Storyboard & secondaire \\
\hline B1.5 & $\begin{array}{l}\text { Le système doit afficher } \\
\text { des alertes de } \\
\text { changement de dernières } \\
\text { minutes. }\end{array}$ & AFE & obligatoire \\
\hline B3 & \multicolumn{3}{|l|}{ Interfaces } \\
\hline B3.1 & $\begin{array}{l}\text { Le système doit fournir } \\
\text { une identification d'un } \\
\text { utilisateur }\end{array}$ & Prototype Idée 2 & obligatoire \\
\hline B3.2 & $\begin{array}{l}\text { Le système doit être } \\
\text { interactif (écran tactile) }\end{array}$ & Prototype Idée 2 & obligatoire \\
\hline
\end{tabular}

Figure 4 : Exemple de synthèse des besoins, avec justification et niveau de priorité

\section{MISE EN CEUVRE DE L'ENSEIGNEMENT}

Afin de remplir les objectifs pédagogiques, nous avons conçu l'architecture suivante (avec une contrainte de nombre d'heures) :

- $2 \mathrm{~h}$ sur les processus d'ingénierie système, les défis du processus de définition des besoins, présentation du projet fil rouge et $\mathrm{du}$ document d'expression des besoins ;

- 6h de conception participative : techniques d'interview in-situ, scénarios de travail, brainstormings oral et vidéo, scénarios de conception et design walkthrough [24];

- 4h d'analyse fonctionnelle, avec la méthode APTE ;

- 6h d'approche orientée objet: le modèle du domaine, les cas d'utilisation et les diagrammes de séquence ;

- 4h sur la rédaction et la synthèse des besoins, le passage aux exigences, et la rédaction du document d'expression des besoins.

Il est demandé aux étudiants par groupes de quatre de conduire l'étude de besoins sur un projet fil rouge en appliquant au fur et à mesure des cours les techniques enseignées, avec pour but de rédiger le document d'expression des besoins. Le projet fil rouge consiste en une demande de l'ENAC pour des améliorations de son système de consultation des emplois du temps, afin notamment de proposer les emplois du temps sur Smartphone. Le choix du système de l'emploi du temps comme objet d'étude nous permet d'avoir une multitude d'utilisateurs à disposition, entre les étudiants, les enseignants et l'administration.

Nous sommes conscients que le sujet proposé a des limites. Par exemple, les étudiants, étant eux-mêmes utilisateurs de l'emploi du temps, pourraient tomber dans le piège de concevoir pour leurs seuls besoins. Nous aurions pu choisir un système avec des utilisateurs n'étant pas les concepteurs. Cependant, ce sujet étant proposé pour l'ensemble d'une promotion, il est difficilement concevable qu'une multitude de groupes d'étudiants recueillent des besoins avec des interviews auprès d'un ensemble restreint d'utilisateurs, et ceci chaque année ! Il faut donc que le réservoir d'utilisateurs change régulièrement pour éviter la lassitude des interviewés, et que ce réservoir soit grand car les interviewers sont nombreux. Par ailleurs, l'emploi du temps est utilisé par d'autres étudiants qui ont des besoins différents de ceux des étudiants concernés par le cours (stagiaire sur une semaine, formations très spécifiques avec des équipements dédiés etc.). Aussi, les étudiants formés doivent quand même récolter des besoins qui ne correspondent pas à leur usage. Par ailleurs, même si un système d'emploi du temps est complexe à concevoir et à réaliser, la complexité n'est sans doute pas aussi grande que celles des systèmes que les futurs diplômés sont amenés à concevoir. Nous limitons la complexité du problème pour deux raisons. Tout d'abord, la durée ainsi que la charge de travail dévolue à ce cours sont limitées. De plus, notre cours est axé sur l'acquisition de compétences concernant la mise en 
application de plusieurs techniques. Nous avons estimé qu'une étape d'acquisition était nécessaire et qu'aborder la gestion de la complexité durant cette étape lui serait peutêtre nuisible. L'étape complémentaire de gestion de complexité est progressivement prise en charge dans la suite du cursus, par les projets java, les projets de synthèse, plus longs, et les stages en entreprise.

\section{RETOUR D'EXPÉRIENCE}

L'enseignement est mis en application par les élèves à travers le projet fil rouge décrit dans la section précédente. Il l'est aussi à travers un projet de conception et de programmation dit «projet java». Sans que cela soit son seul objectif, ce projet constitue néanmoins une première mise en pratique de l'enseignement sur un développement de système de bout en bout durant deux mois et demi. Nous avons interviewé cinq clients ayant proposé un projet java avec comme objectifs :

- d'évaluer l'application des techniques enseignées à bon escient selon les projets ;

- de récolter un retour sur la satisfaction des clients par rapport à leurs attentes sur le projet ;

- d'estimer s'il y a une probabilité plus élevée d'application des techniques enseignées dans l'industrie.

Du fait d'être réalisés auprès des clients, ces interviews apportent une vision "de l'intérieur" sur la dynamique des projets dont les auteurs de cet article, enseignants, n'ont souvent vu que le résultat final. Certains des clients sont aussi enseignants et de ce fait, sont informés sur le contenu du cours d'IS et les techniques enseignées, d'autres non. Les entretiens, de type ouvert, d'une durée de $30-45 \mathrm{mn}$, ont été partiellement ou complètement transcrits et portaient sur les projets des années 2011, 2012 et 2013. $\mathrm{Au}$ cours de ces entretiens nous avons pu amorcer une évaluation des exigences formulées pour l'enseignement que nous proposons et rappelées par la Figure 2.

\section{Susciter la co-évolution besoin-solution}

Les éléments issus des interviews des clients se rapportant à cette exigence sont essentiellement les techniques itératives s'appuyant sur le prototypage.

- itérations : les projets ont en général donné lieu à deux itérations au plus, en fin de projet;

- l'un des clients regrettait que les élèves n'abordent pas le codage assez tôt ; c'était le cas pour un projet nécessitant de gérer le risque de difficulté d'intégration dans un environnement technique de gestion de drones par des expérimentations organisées assez tôt dans le projet ;

- en revanche, pour un projet de modélisation 3D d'avion, les deux tiers du temps imparti avaient été consacrés à la spécification des besoins, suivis d'une phase de codage très rapide mais d'autant plus efficace que les besoins étaient bien analysés et le maquettage productif, le client a souligné cette rapidité : « c'était comme s'il n'y avait plus qu'à » (coder);
- à noter un cas intéressant d'application d'une démarche dite de design créatif [14] différente de celle enseignée mais réussie, au cours de laquelle, pour un projet de simulateur pour le cockpit, les élèves ont surtout codé mais aussi exploré et innové au-delà de ce qu'on leur demandait en itérant auprès d'un utilisateur ;

- prototypage: tous les projets n'ont pas fait appel au prototypage basse et moyenne fidélité, d'autant plus que cela n'était pas obligatoire :

- certains élèves dont le projet était prédécoupé en petits sous projets bien définis par le client (des instruments pour un simulateur de cockpit) ont préféré du prototypage informatique direct ;

- d'autres ont réalisé des prototypes basse-fidélité, mais semble-t-il plutôt sur demande de leur client ;

- un client a mentionné la préférence des élèves pour la discussion à leur avis plus rapide que le maquettage ;

- $\quad$ selon ce même client, les élèves s'auto-censurent dès que les fonctions ou les interactions à concevoir semblent trop compliquées à coder ;

- $\quad$ pour le groupe de modélisation 3D d'avion qui avait proposé plusieurs itérations de maquettage, le tuteur en a apprécié leur aspect concret et précis, permettant d'effectuer des choix sur des propositions spécifiques et répondant en général très bien aux besoins identifiés.

\section{Définir de façon exhaustive les caractéristiques techniques du système}

Les sujets proposés dans le cadre de ces projets ne concernent pas des systèmes complexes à proprement parler. Il est donc difficile d'évaluer l'atteinte de cette exigence, d'autant que cela n'est pas demandé dans le cadre du projet java. On peut cependant noter que l'utilisation de la méthode APTE permet de systématiser la caractérisation des performances du système (en termes de temps de calcul, temps d'affichage, nombre d'actions utilisateur, etc.), ce qui était auparavant non traité par les élèves.

\section{Définir l'usage}

De manière générale, les tuteurs se sont montrés très satisfaits de la compréhension et de la spécification des besoins des utilisateurs par les élèves. L'approche est ainsi qualifiée de très "confortable» sur ce point par le client du projet de modélisation 3D d'un avion. Il faut préciser que sur l'aspect de définition de l'usage, les projets s'avèrent toutefois assez différents. Certains sont très définis, le client privilégiant le travail de programmation et proposant des interactions volontairement spécifiées de manière très précise, comme dans le cas d'un projet «Google Earth». D'autres projets sont complètement ouverts, comme des projets autour de systèmes à particules ou d'interacteurs tangibles qui laissaient aux élèves des choix importants sur les interactions. 
Nous pouvons également mentionner les retours des clients face à l'utilisation des scénarios. Les scénarios de travail ou les scénarios de conception sont plutôt bien utilisés en général, que ce soit pour guider le maquettage, ou pour guider la conception par objets. Certains élèves ont parfois éprouvé une difficulté ou une incompréhension dans leur utilisation :

- $\quad$ selon le client du projet de simulateur de vol, les scénarios de conception sont "triviaux" car ils ne présentent qu'une seule interaction avec le système ("lancer la simulation", "afficher") : par crainte de détailler ces scénarios à un niveau trop concret (proche de l'implémentation), les élèves préfèrent ne pas les rédiger ;

- l'un des clients a constaté que les élèves n'opéraient aucun saut innovant entre les scénarios de travail (issus des observations sur le terrain) et les scénarios de conception : par exemple, l'observation d'une sélection en rouge sur l'ancien système "papier" avait résulté directement en sélection en rouge dans le système informatique proposé à travers un scénario de conception; ce problème soulève la question de la compréhension de la démarche d'innovation; [4] remarque à ce titre que certaines mésinterprétations de la démarche poussent à croire (à tort) que les observations de l'existant dictent les choix pour le système futur.

\section{Formaliser}

La rédaction d'une synthèse des besoins (cf. figure 4) est réalisée par l'ensemble des groupes, car demandée dans le cadre du projet java. Elle a permis de structurer les échanges avec les clients, notamment pour identifier les priorités en termes de codage. En ce qui concerne l'analyse orientée objet, les clients ne maîtrisent pas nécessairement ce type d'analyse, et le plus souvent ne prennent pas spontanément connaissance des modélisations produites par les élèves, mais les élèves les consultent parfois pour valider leurs schémas. Ainsi, l'un d'eux raconte que le «diagramme de création d'objets » semblait permettre aux élèves 1) de mieux comprendre ce qui reliait les différents éléments d'information, et 2) de poser des questions pour savoir si « des données ou des paramètres étaient spécifiques à un objet en particulier ou est-ce qu'il devait être détaché de l'objet », avérant ainsi le rôle joué par les modèles objets pour la communication et la compréhension de l'activité.

\section{Discussion}

Ces retours d'expérience sont préliminaires mais donnent des premiers éléments sur les points qui fonctionnent et ceux à améliorer. De manière générale, le processus enseigné est relativement bien assimilé. Selon certains clients qui encadrent des projets d'élèves n'ayant pas suivi la formation, l'approche inscrit les étudiants dans une dynamique de proposition; elle les rend particulièrement autonomes et, dans la mesure où le client parvient à «jouer le jeu » du client ou de l'utilisateur, cette démarche fait sortir les élèves du cadre scolaire. Certaines des techniques enseignées ne s'appliquent pas à tous les projets, selon le cahier des charges fourni en entrée : ainsi le prototypage basse-fidélité ou les itérations sont plutôt utilisés dans les projets comportant une part de risque et d'incertitude, ou bien le brainstorming qui sera généralement utilisé pour les projets ouverts en terme d'interactions envisageables. L'application partielle des techniques enseignées est également prévisible dans le cadre de projets mettant l'accent sur la programmation. Les projets java constituent une première mise en situation sur des sujets qui, certes, ne peuvent pas être représentatifs des systèmes complexes, mais qui restent le plus souvent réalistes d'un point de vue industriel.

\section{CONCLUSION ET PERSPECTIVES}

A partir de l'analyse des problèmes des pratiques industrielles et de nos enseignements existants, nous avons dégagé le besoin d'un nouvel enseignement en ingénierie des systèmes homme-machine complexes, selon quatre exigences dimensionnantes: définir l'usage, décrire de façon exhaustive les caractéristiques techniques, susciter la co-évolution besoin-solution, formaliser les besoins. Trois disciplines sont traditionnellement enseignées en école d'ingénieurs pour répondre à ces exigences : l'analyse fonctionnelle, la conception participative et l'approche orientée objet. L'identification des points forts et des limites de chacune de ces disciplines nous a conduit à envisager de façon pragmatique une combinaison des techniques issues de ces disciplines, en s'efforçant de les intégrer dans les processus d'ingénierie système largement utilisés dans l'industrie. Le résultat de la conduite du processus et de l'application des techniques est la production d'un document d'expression des besoins.

La mise en application par les étudiants sur les projets de fin de deuxième année et de troisième année montrent cependant un manque de recul sur ces techniques : il reste difficile pour eux de discerner les techniques les plus appropriées à mettre en œuvre face à un cahier des charges. Pour cela, nous souhaitons développer leur sens critique [26] en incluant un nouvel objectif pédagogique dans notre cours et en leur fournissant des pistes d'analyse : comment détecter qu'un cahier des charges est bien formulé et permet d'entamer le travail de spécification et de conception ? Comment détecter les problèmes ? Comment analyser ces problèmes et $\mathrm{y}$ répondre ? Nous souhaiterions enfin mieux intégrer le processus de design créatif, essentiel en développement de systèmes interactifs, au processus IS, en poursuivant une réflexion très présente dans la communauté IHM [14][37].

\section{REFERENCES}

1. AFNOR X50-151. Analyse de la valeur, analyse fonctionnelle - Expression fonctionnelle du besoin et cahier des charges fonctionnel. 1991

2. Barthet M. F. et Tarby J. C. The Diane+ method. In Computer-aided design of user interfaces, pages 95120, Namur, Belgium, 1996. 
3. Beyer H. and Holtzblatt K. Contextual design. interactions 6, 1 (January 1999), 32-42.

4. Bødker S. and Sejer Iversen O. Staging a professional participatory design practice: moving PD beyond the initial fascination of user involvement. In Proc. of NordiCHI '02. ACM (2002), 11-18

5. Boehm B. "Some Future Trends and Implications for Systems and Software Engineering Processes." Systems Engineering 9, 1 (Spring 2006): 1-19.

6. Bourguin, G., Derycke, A., Tarby, J. C. Beyond the Interface: Co-evolution inside Interactive Systems A Proposal Founded on Activity Theory, In Proc. of IHM-HCI, 2001

7. Bytheway C. W. Genesis of FAST. Value World, 28. (2005).

8. Carroll, J.M., Rosson, M.B. Deliberated Evolution: Stalking the View Matcher in design space. HumanComputer Interaction, 6 (3 and 4), 1992, 281-318.

9. Christensen M., Crabtree A., Heide Damm C., Hansen K. M., Lehrmann Madsen O., Marqvardsen P., Mogensen P., Sandvad E., Sloth L. and Thomsen M. The M.A.D. Experience: Multiperspective Application Development in evolutionary prototyping. In Proc. of ECCOP '98. Springer-Verlag (1998), 13-40.

10. Coad P. and Yourdon E. Object-Oriented Analysis (2nd Ed.). Yourdon Press. (1991).

11. Constantine L., Biddle R. and Noble J. Usagecentered design and software engineering: Models for integration. In Proc. of ICSE 2003, ICSE Workshop on SE-HCI, pages 106-113, 2003.

12. Dahlbom, B. and Mathiassen, L. (1993). Computers in Context: The Philosophy and Practice of Systems Design (Oxford: Blackwell).

13. de la Bretsche B. La méthode APTE : Analyse de la valeur, analyse fonctionnelle, Pétrelle, 2000.

14. Fallman D. Design-oriented human-computer interaction. In Proc of $C H I$ '03. (2003). ACM, 225-232.

15. Graduate Reference Curriculum for System Engineering (GRCSE) - 0.5 - Body of Knowledge and Curriculum to Advance Systems Engineering (BKCASE) - Stevens Institute of Technology - 2011

16. Graduate Software Engineering 2009 (GSwE2009) Curriculum Guidelines for Graduate Degree Programs in Software Engineering. Stevens Institute of Technology.

17. INCOSE. System Engineering Handbook. V3.2.2, 2011.

18. ISO IEC 15288-2008. System engineering and software engineering - System life cycle processes. IEEE.

19. ISO 9241-210 Ergonomie de l'interaction hommesystème -- Partie 210: Conception centrée sur l'opérateur humain pour les systèmes interactifs. Organisation internationale de normalisation.
20. ITU. Recommendation Z.151 (GRL) V3.0, 2003.

21. Luzeaux D., Ruault R., Wippler J-L. Maitrise de l'ingénierie des systèmes complexes et des systèmes de systèmes, étude de cas. Hermès (2011).

22. Larman C. Applying UML and Patterns: An Introduction to Object-Oriented Analysis and Design and Iterative Development (3rd Edition). (2004). Prentice Hall PTR, Upper Saddle River, NJ, USA.

23. Mackay, W.E. Beyond iterative design: User innovation in co-adaptive systems. (Technical Report 1991-1305) Rank Xerox EuroPARC, Cambridge, England. (1992)

24. Mackay, W.E. Video to Support Interaction Design, DVD, ISBN 1-58113-516-5, ACM, New York, 2002.

25. Mackay, W.E. Educating Multi-disciplinary Design Teams. In Proc. of Tales of the Disappearing Computer, Santorini, Greece, (2003), pp 105-118.

26. Mergendoller, J. R., Maxwell, N. L., \& Bellisimo, Y. The effectiveness of problem-based instruction: A comparative study of instructional methods and student characteristics. The Interdisciplinary Journal of Problem-based Learning, 1(2), 49-69, (2006).

27. Meyer B. Object-Oriented Software Construction (2nd Ed.). Prentice-Hall, Inc., Upper Saddle River, NJ, USA (1997).

28. Micouin P. Proposition pour la définition et la mise en place de processus d'ingénierie système : application au cas de la conception concourante dans le secteur automobile. Thèse de doctorat. ENSAM. 2006

29. Muller, M.J and Kuhn, S. Participatory design. Commun. ACM 36, 6 (June 1993), 24-28.

30. Norman D. A. Human-centered design considered harmful. interactions 12, 4 (Jul 2005), 14-19.

31. Roques P. UML 2 par la pratique. (2009). Eyrolles.

32. Ross D. T. Structured Analysis (SA): A Language for Communicating Ideas. IEEE Transactions on Software Engineering, SE-3(1), pp. 16-34.

33. Sousa K. et Furtado E. An approach to integrate hci and se in requirements engineering. In Proc. of the IFIP TC13 workshop on Closing the gaps: Software engineering and Human-Computer Interaction, 2003.

34. Standish Group. CHAOS report: project success / failure factors - Standish Group Chaos report - 2009.

35. Trygve M. H. Reenskaug. Models - Views Controllers, Xerox PARC technical note, Dec. 1979. heim.ifi.uio.no/ trygver/1979/mvc-2/1979-12-MVC.pdf

36. Van Lamsweerde A. Goal-Oriented Requirements Engineering: A Guided Tour. Invited Paper for RE'01 - 5th IEEE International Symposium on Requirements Engineering, Toronto, August, 2001, pp. 249-263.

37. Vetting Wolf T., Rode J. A., Sussman J., and Kellogg W. A. Dispelling "design" as the black art of CHI. In Proc. of CHI '06. (2006). ACM, 521-530 